\title{
Long-Term Evaluation of Nasal Septoplasty Followed by Inferior Turbinate Cauterization for the Treatment of Nasal Obstruction Using Objective and Subjective Methods
}

\author{
Konstantinos Valsamidis ${ }^{1} \quad$ Konstantinos Titelis $^{1}$ \\ Konstantinos Markou ${ }^{3}$ Stefanos Triaridis ${ }^{2}$ \\ ${ }^{1}$ Department of Otorhinolaryngology, Georgios Gennimatas General \\ Hospital, Thessaloniki, Greece \\ 21 st University Department of Otorhinolaryngology, AHEPA \\ University Hospital, Aristotle University of Thessaloniki, \\ Thessaloniki, Greece \\ 3 2nd University Department of Otorhinolaryngology, Geniko \\ Nosokomeio Thessalonikis Papageorgiou, Thessaloniki, Greece
}

\begin{abstract}
Address for correspondence Konstantinos Valsamidis, MD, MSc, Department of Otorhinolaryngology, Georgios Gennimatas General Hospital, Ethinikis Amynis 41 Thessaloniki 54635, Greece (e-mail: kosvals@hotmail.com).
\end{abstract}

Int Arch Otorhinolaryngol 2018;22:284-290.

\begin{abstract}
Keywords

- nasal obstruction

- septoplasty

- acoustic rhinometry

- quality of life
\end{abstract}

Introduction Nasal septoplasty is considered the treatment of choice for nasal obstruction due to septal deviation. An ongoing discussion among rhinologists is whether it is reasonable to perform objective measurements of nasal patency pre or postoperatively routinely.

Objective The primary aim of this study was to identify the short- and long-term functional benefits for patients undergoing septal surgery, as assessed by acoustic rhinometry (AR). The secondary goal was to evaluate the short- and long-term perception of symptom relief and disease-specific quality of life (QoL) outcomes on the part of the patients.

Methods This was a prospective observational study in which AR was utilized for the assessment of nasal patency preoperatively and 1, 6 and 36 months after septoplasty. Total 40 patients who underwent septoplasty filled out the Nasal Obstruction Septoplasty Effectiveness (NOSE) questionnaire and the Glasgow Benefit Inventory (GBI) to assess their subjective improvement in nasal obstruction symptoms and the changes in their QoL.

Results There were statistically significant improvements in nasal patency, mean postoperative NOSE and GBI scores postoperatively. However, there was no correlation between the mean NOSE and GBI scores and the AR measurements. Furthermore, the $G B I$ scores tended to decrease as the postoperative period increased.

Conclusion The present study confirms that septoplasty significantly increases nasal patency and causes a significant subjective improvement in nasal obstruction symptoms. The absence of a statistically significant correlation among the objective measurements, the symptom scores, and the patients' low GBI scores indicates that factors other than the anatomical findings may also contribute to the patients' perception of QoL. received

April 30, 2017

accepted

September 30, 2017

published online

January 18,2018
Copyright (e) 2018 by Thieme Revinter

Publicações Ltda, Rio de Janeiro, Brazil
License terms

10.1055/s-0037-1613688. ISSN $1809-9777$. c) $(1) \$$ 


\section{Introduction}

In patients with nasal and sinus disease, nasal obstruction is the most common complaint. ${ }^{1}$ Deviation of the nasal septum is one of the most frequent causes of nasal obstruction, and nasal septoplasty is considered the definitive treatment for septal deviation. 2,3 As the symptoms do not necessary correlate to the clinical findings, criteria for the objective assessment of nasal airway patency are required for an accurate diagnosis, an appropriate therapy, and an evaluation of the results. The gold standard would be a reproducible, quantifiable, objective test with a strong correlation to the subjective perception of nasal airflow. Considering the complexity and variability of the subjective feeling of nasal airway patency, one may reasonably wonder whether such a test will ever be available. ${ }^{4}$ Additionally, health care provision has become increasingly shaped by the patients' needs and preferences. In recent years, a shift in emphasis toward patient-oriented outcome measures is evident. ${ }^{5-11}$ Several previous studies report on subjective and objective outcome measures after septoplasty. However, most of them were retrospective or based on the physicians' clinical findings and not on patients' subjective symptoms, and did not investigate the long-term results of nasal septoplasty. 1,2,5-12

The primary aim of this study is to identify any long-term functional benefits of nasal septal surgery, by providing evidence of a change in nasal airway patency as assessed by the objective method of acoustic rhinometry (AR). Moreover, we evaluated the patients' perception of the surgical benefits by measuring the impact of the procedure on their diseasespecific quality of life (QoL) shortly as well as long after surgery.

\section{Materials and Methods}

A prospective observational study was performed with 40 consecutive patients presenting to a district general hospital for septoplasty during a 6-month period (from December 2013 to June 2014). All patients included in the study were followed-up for at least three years and were available for statistical analysis. According to the inclusion criteria, we enrolled patients aged $\geq 18$ years with chronic nasal obstruction (lasting for at least 6 months) and nasal septum deviation upon clinical examination. The symptom of nasal obstruction should be persistent after a four-week trial of medical management that includes topical nasal steroids, topical or oral decongestants, or an oral antihistamine/ decongestant combination. We excluded from this study patients: with a history of sinonasal malignancy, history or clinical evidence of chronic sinusitis, allergic rhinitis, a history of radiation therapy to the head and neck, acute nasal trauma or fracture in the past three months, septal perforation, craniofacial syndrome, nasal valve collapse, adenoid hypertrophy, Wegener granulomatosis, sarcoidosis, uncontrolled asthma, those pregnant, and those who had undergone other ENT procedures such as rhinoplasty and sinus surgery concomitant to septal surgery. All patients who met the eligibility criteria and agreed to participate gave signed informed consent and were enrolled in the study. The study protocol was approved by the institutional Review Board (under no 273 /01.10.2013).

The patients were classified into three groups based on the severity of the septum deviation: a) grade I: mild deviation less than half the total distance to the lateral nasal wall; b) grade II: moderate deviation - more than half the distance but not touching the lateral nasal wall; and c) grade III: severe deviation - deviation touching the lateral nasal wall. This classification was proposed by Jin et al. ${ }^{13}$ The patients were asked about their symptoms and disease-specific QoL. In order to assess these outcomes, we utilized two previously published questionnaires, which were translated and validated into the Greek language and used in accordance with previously published studies (The Nasal Obstruction Septoplasty Effectiveness [NOSE] scale, which is a validated scale to measure nasal obstruction symptoms, and the Glasgow Benefit Inventory (GBI), which is an ear, nose and throat [ENT] post-intervention questionnaire). The GBI consists of three subscales that assess the influence of the surgery on the patients' physical health, psychosocial function, and social interaction. ${ }^{14,15}$

Minimal cross-sectional areas (MCAs) at the first $2 \mathrm{~cm}$ (MCA1) and between 2-5 cm (MCA2) into the nasal cavity were recorded for the deviated side of nose with the use of AR.

The operation technique was fixed and included a hemitransfixion incision followed by an elevation of the septal mucoperichondrium in one or both sides, addressing all areas of deviation, reshaping and/or removing the deviated part of the cartilage. All patients also underwent a cauterization of the inferior nasal turbinate that was intended to reduce its size. All patients were followed-up for a clinical assessment (ENT examination, AR) and filled out the NOSE and GBI questionnaires 1, 6 and 36 months after surgery. All surgeries were performed by the same consultant surgeon, who was blinded to the patients' NOSE and GBI scores both before and after the treatment, and did not participate in the collection of the questionnaires. Another clinician was in charge of the data collection.

\section{Statistical Analysis}

The variables studied are presented as means with standard deviation. The study required 40 patients in order to have a two-tailed significance test, a significance level of 0.05 and a power $>85 \%$ to detect a difference as small as 0.05 among the means of the compared groups, for a standard deviation of 0.1 . After assessing the patients for normality with the Shapiro-Wilk test, we used the independent sample $t$-test and the paired sample $t$-test for the group comparison of the data with normal distribution. When the data did not have normal distribution, the Mann-Whitney $U$ test and the Wilcoxon $t$-test were used. The Kruskal-Wallis test was used for the comparisons of data among more than two groups. Spearman's (rho) correlation coefficient was used to check the correlations of the data. In agreement with other studies, ${ }^{2}$ we used as criteria to measure the success of the operation the median postoperative MCA1 and MCA2 values, as well as the NOSE score. The patients were then divided into two groups (above and below the criteria, which 
consisted of the patients with more and less successful results respectively). The analysis of the GBI scores was based on the comparison of these two groups. A $p$-value $<0.05$ was accepted as the statistical significance level.

\section{Results}

According to the sample size calculation, 40 patients who met the eligibility criteria were included in the study. At the end of the data collection period, all 40 patients (100\%) filled out their follow-up surveys and were available for data analysis. Their mean age was 31.5 (range $18-55$ ) years. Total 25 patients $(62.5 \%)$ were male, and $15(37.5 \%)$ were female.

The results of the AR were evaluated in the deviated side. Regarding the postoperative MCA1 and MCA2 values of the deviated side at 1, 6 and 36 months, they increased when compared with the preoperative values ( - Table 1 ). The patients had significant improvements in their nasal obstruction symptoms after surgery. There was a very significant improvement in the NOSE score 1, 6 and 36 months postoperatively. However, the NOSE score 3 years after surgery was statistically significantly higher than the same score at 6 months, indicating a mild worsening of the nasal symptoms ( - Table 2). Additionally, the GBI scores (total and subscales) of the patients in the current study increased between the 1 st and 6 th months after surgery with statistical significance. However, we should note that these scores were not very high $(<32 / 100)$. Furthermore, there was a statistically significant decrease in GBI scores 6 and 36 months postoperatively for the total as well as for the general and physical health subscale scores ( - Table 3 ).

In all three follow-up periods, there was no correlation between the objective measurements (MCA1 and MCA2 values) and the changes in the subjective symptoms (NOSE scores), and the objective measurements and the patients' QoL (GBI scores; - Table 4). Finally, three years after surgery, the patients were divided into two groups on three different settings (above and below the criteria groups - based on the median postoperative of the three variables: MCA1 and MCA2 values and NOSE score), consisting of patients with more and less successful results respectively. There was a trend for the patients in the above the criteria group (for all the settings) to have total GBI scores higher than the patients in the below the criteria group. Better QoL was specifically recorded for patients with higher postoperative MCA1 and

Table 1 Pre and postoperative (at 1, 6, 36 months after surgery) minimal cross-sectional area (MCA1 and MCA2) measurement with acoustic rhinometry

\begin{tabular}{|c|c|c|c|c|c|c|c|c|c|}
\hline Variable & $\begin{array}{l}\text { Preop } \\
\text { mean (SD) }\end{array}$ & $\begin{array}{l}\text { Postop1 } \\
\text { mean (SD) }\end{array}$ & $p^{\mathrm{a}}$ & $\begin{array}{l}\text { Preop } \\
\text { mean (SD) }\end{array}$ & $\begin{array}{l}\text { Postop6 } \\
\text { mean (SD) }\end{array}$ & $p^{a}$ & $\begin{array}{l}\text { Preop } \\
\text { mean (SD) }\end{array}$ & $\begin{array}{l}\text { Postop36 } \\
\text { mean (SD) }\end{array}$ & $p^{a}$ \\
\hline $\operatorname{MCA} 1\left(\mathrm{~cm}^{2}\right)$ & $0.49 \pm 0.15$ & $0.83 \pm 0.60$ & 0.000 & $0.49 \pm 0.15$ & $0.90 \pm 0.08$ & 0.000 & $0.49 \pm 0.15$ & $0.85 \pm 0.13$ & 0.000 \\
\hline MCA2 $\left(\mathrm{cm}^{2}\right)$ & $0.47 \pm 0.15$ & $1.12 \pm 0.49$ & 0.000 & $0.47 \pm 0.15$ & $1.29 \pm 0.47$ & 0.000 & $0.47 \pm 0.15$ & $1.15 \pm 0.47$ & 0.000 \\
\hline
\end{tabular}

Abbreviations: MCA1, minimal cross-sectional area at the first $2 \mathrm{~cm}$ of the nasal cavity; MCA2, minimal cross-sectional area between 2-5 cm into the nasal cavity; Preop, preoperatively; Postop1, 1 month postoperatively; Postop6, 6 months postoperatively; Postopo36, 36 months postoperatively; SD, standard deviation.

Note: ${ }^{a}$ Wilcoxon signed-rank test for paired samples.

Table 2 Pre and postoperative (at 1, 6, 36 months after surgery) NOSE scores

\begin{tabular}{|l|l|l|l|l|l|l|}
\hline Variable & Preop mean (SD) & Postop1 mean (SD) & $p^{\mathrm{a}}$ & Preop mean (SD) & Postop6 mean (SD) & $p^{\mathrm{a}}$ \\
\hline NOSE & $79.12 \pm 15.05$ & $19.87 \pm 15.08$ & 0.000 & $79.12 \pm 15.05$ & $8.87 \pm 8.35$ & 0.000 \\
\hline & Preop mean (SD) & Postop36 mean (SD) & $p^{\mathrm{a}}$ & Postop6 mean (SD) & Postop36 mean (SD) & $p^{\mathrm{a}}$ \\
\hline NOSE & $79.12 \pm 15.05$ & $15.12 \pm 8.95$ & 0.000 & $8.87 \pm 8.35$ & $15.12 \pm 8.95$ & 0.000 \\
\hline
\end{tabular}

Abbreviations: NOSE, Nasal Obstruction Septoplasty Effectiveness Questionnaire; Preop, preoperatively; Postop1, 1 month postoperatively; Postop6, 6 months postoperatively; Postop36, 36 months postoperatively; SD, standard deviation.

Note: ${ }^{a}$ Wilcoxon signed-rank test for paired samples.

Table 3 Postoperative (at 1, 6, 36 months after surgery) GBI scores - total and subscales

\begin{tabular}{|l|l|l|l|l|l|l|}
\hline Variable & Postop1 mean (SD) & Postop6 mean (SD) & $p^{\mathrm{a}}$ & Postop6 mean (SD) & Postop36 mean (SD) & $p^{\mathrm{a}}$ \\
\hline GBI total & $22.36 \pm 12.26$ & $25.76 \pm 13.13$ & $0.000^{\mathrm{b}}$ & $25.76 \pm 13.13$ & $19.86 \pm 9.27$ & $0.001^{\mathrm{b}}$ \\
\hline GBI general & $26.04 \pm 15.61$ & $30.31 \pm 15.87$ & $0.000^{\mathrm{b}}$ & $30.31 \pm 15.87$ & $22.49 \pm 12.86$ & $0.000^{\mathrm{b}}$ \\
\hline GBI social & $4.16 \pm 11.78$ & $6.25 \pm 12.33$ & 0.009 & $6.25 \pm 12.33$ & $5.2 \pm 11.43$ & 0.219 \\
\hline GBI physical & $26.24 \pm 22.29$ & $31.24 \pm 22.70$ & 0.02 & $31.24 \pm 22.70$ & $20.83 \pm 22.24$ & 0.003 \\
\hline
\end{tabular}

Abbreviations: GBI, Glasgow Benefit Inventory; Preop, preoperatively; Postop1, 1 month postoperatively; Postop6, 6 months postoperatively; Postop36, 36 months postoperatively; SD, standard deviation.

Notes: ${ }^{a}$ Wilcoxon signed-rank test for paired samples. ${ }^{\mathrm{b}}$ Paired samples $t$-test. 
Table 4 Correlation between MCA1 and MCA2 values and NOSE and GBI scores 36 months postoperatively

\begin{tabular}{|c|c|c|}
\hline & NOSE postop36 & GBI postop36 \\
\hline \multicolumn{3}{|c|}{ MCA1 postop36 } \\
\hline r-value ${ }^{a}$ & -0.085 & 0.114 \\
\hline$p$ & 0.604 & 0.482 \\
\hline \multicolumn{3}{|c|}{ MCA2 postop36 } \\
\hline$r$-value ${ }^{a}$ & -0.125 & 0.085 \\
\hline$p$ & 0.444 & 0.6 \\
\hline
\end{tabular}

Abbreviations: GBI, Glasgow Benefit Inventory; MCA1, minimal cross-sectional area at the first $2 \mathrm{~cm}$ of the nasal cavity; MCA2, minimal cross-sectional area between $2-5 \mathrm{~cm}$ into the nasal cavity; NOSE, Nasal Obstruction Septoplasty Effectiveness Questionnaire.

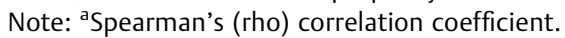

MCA2 values and lower NOSE scores, but in no case the results reached statistical significance (-Table $\mathbf{5}$ ).

Furthermore, six months after surgery, the patients with preoperative mild septum deviation had greater improvement in nasal patency (higher MCA values) and symptom severity (better NOSE scores) and better QoL (higher GBI results). All comparisons among the patients with different degrees of septum deviation showed statistically significant differences (-Table 6). The same results were found three years postoperatively. However, despite the fact that patients with a preoperative mild degree of septum deviation tend to have better QoL, the differences among patients with mild, moderate and severe degrees of septum deviation did not reach statistical significance ( - Table $\mathbf{7}$ ).

\section{Discussion}

In the current prospective study, the short- and long-term benefits from nasal septoplasty for the patients were evaluated by using objective and subjective methods. We found that $90 \%$ of the patients with nasal obstruction and septal deformity who underwent nasal septoplasty had significant improvements (better NOSE scores) in nasal obstruction in the postoperative short-term follow-up. This result was sustained 3 years postoperatively but in a smaller degree, as $75 \%$ of the patients reported they were free from the symptoms of nasal obstruction.

Outcome researches regarding the long-term results of septoplasty are not new. However, there are some major differences between the previous studies and the present one. First of all, some previous studies were retrospective, ${ }^{16-19}$ while others used non-validated disease-specific questionnaires. ${ }^{17,20}$ In their retrospective study, Sundh and Sunnergren used a questionnaire based on the Swedish National Quality Registry for Septoplasty. The main finding was that more than half of the patients (53\%) reported that their symptoms remained or had worsened 3 to 6 years after septoplasty. Another important finding was that $83 \%$ of the patients reported they still suffered from nasal obstruction at the long-term follow-up. Additionally, the degree of symptom

Table 5 Comparison of postoperative (36 months after surgery) total GBI scores between the groups above below criterion A (median MCA1 value), criterion B (median MCA2 value) and criterion C (median value of the NOSE score)

\begin{tabular}{|l|l|l|l|l|l|l|l|l|l|}
\hline & \multicolumn{3}{|l|}{ Criterion A } & \multicolumn{3}{l|}{ Criterion B } & \multicolumn{2}{l|}{ Criterion C } \\
& $\begin{array}{l}\text { Above } \\
(N=22)\end{array}$ & $\begin{array}{l}\text { Below } \\
(N=18)\end{array}$ & & $\begin{array}{l}\text { Above } \\
(N=22)\end{array}$ & $\begin{array}{l}\text { Below } \\
(N=18)\end{array}$ & & $\begin{array}{l}\text { Above } \\
(N=17)\end{array}$ & $\begin{array}{l}\text { Below } \\
(N=23)\end{array}$ & \\
\hline Variable & Mean (SD) & Mean (SD) & $\boldsymbol{p}^{*}$ & Mean (SD) & Mean (SD) & $\boldsymbol{p}^{*}$ & Mean (SD) & Mean (SD) & $\boldsymbol{p}^{*}$ \\
\hline GBI postop36 & 20.64 & 20.33 & 0.934 & 21.78 & 19.45 & 0.528 & 23.56 & 18.24 & 0.151 \\
& $(10.03)$ & $(8.54)$ & & $(9.28)$ & $(9.44)$ & & $(9.16)$ & $(9.23)$ & \\
\hline
\end{tabular}

Abbreviations: GBI, Glasgow Benefit Inventory; MCA1, minimal cross-sectional area at the first $2 \mathrm{~cm}$ of the nasal cavity; MCA2, minimal cross-sectional area between $2-5 \mathrm{~cm}$ into the nasal cavity; NOSE, Nasal Obstruction Septoplasty Effectiveness Questionnaire; postop36, 36 months postoperatively; SD, standard deviation.

Note: ${ }^{a}$ Mann-Whitney $\mathrm{U}$ test.

Table 6 Comparison of postoperative (at 6 and 36 months after surgery) minimal cross-sectional area (MCA1 and MCA2) measurement with acoustic rhinometry among the groups with different degrees of septum deviation

\begin{tabular}{|c|c|c|c|c|c|c|c|c|}
\hline Variable & $\begin{array}{l}\text { MCA1 } \\
\text { postop6 } \\
\text { mean (SD) }\end{array}$ & $p^{a}$ & $\begin{array}{l}\text { MCA2 } \\
\text { postop6 } \\
\text { mean (SD) }\end{array}$ & $p$ & $\begin{array}{l}\text { MCA1 } \\
\text { postop36 } \\
\text { mean (SD) }\end{array}$ & $p$ & $\begin{array}{l}\text { MCA2 } \\
\text { postop36 } \\
\text { mean (SD) }\end{array}$ & $p$ \\
\hline Grade I $(N=19)$ & $0.86 \pm 0.05$ & \multirow[t]{3}{*}{0.000} & $1.36 \pm 0.59$ & \multirow[t]{3}{*}{0.000} & $0.93 \pm 0.15$ & \multirow[t]{3}{*}{0.03} & $1.31 \pm 0.52$ & \multirow[t]{3}{*}{0.04} \\
\hline Grade II $(N=13)$ & $0.77 \pm 0.35$ & & $0.9 \pm 0.09$ & & $0.82 \pm 0.11$ & & $1.10 \pm 0.41$ & \\
\hline Grade III $(N=8)$ & $0.69 \pm 0.08$ & & $0.69 \pm 0.1$ & & $0.76 \pm 0.17$ & & $0.86 \pm 0.12$ & \\
\hline
\end{tabular}

Abbreviations: MCA1, minimal cross-sectional area at the first $2 \mathrm{~cm}$ of the nasal cavity; MCA2, minimal cross sectional area between 2-5 $\mathrm{cm}$ into the nasal cavity; postop6, 6 months postoperatively; postopo36, 36 months postoperatively; SD, standard deviation.

Note: ${ }^{a}$ Kruskal-Wallis test. 
Table 7 Comparison of postoperative (at 6 and 36 months after surgery) NOSE and GBI scores among the groups with different degrees of septum deviation

\begin{tabular}{|c|c|c|c|c|c|c|c|c|}
\hline Variable & $\begin{array}{l}\text { NOSE } \\
\text { postop6 } \\
\text { mean (SD) }\end{array}$ & $p^{a}$ & $\begin{array}{l}\text { GBI } \\
\text { postop6 } \\
\text { mean (SD) }\end{array}$ & $p$ & $\begin{array}{l}\text { NOSE } \\
\text { Postop36 } \\
\text { mean (SD) }\end{array}$ & $p$ & $\begin{array}{l}\text { GBI } \\
\text { postop36 } \\
\text { mean (SD) }\end{array}$ & $p$ \\
\hline Grade I $(N=19)$ & $12.63 \pm 11.34$ & \multirow[t]{3}{*}{0.000} & $32.74 \pm 8.61$ & \multirow[t]{3}{*}{0.001} & $10.26 \pm 3.52$ & \multirow[t]{3}{*}{0.001} & $22.95 \pm 8.72$ & \multirow[t]{3}{*}{0.119} \\
\hline Grade II $(N=13)$ & $20.26 \pm 7.58$ & & $15.59 \pm 4.47$ & & $16.92 \pm 9.9$ & & $18.37 \pm 9.03$ & \\
\hline Grade III $(N=8)$ & $45 \pm 5.34$ & & $8.68 \pm 4.55$ & & $23.12 \pm 10.32$ & & $14.93 \pm 9.26$ & \\
\hline
\end{tabular}

Abbreviations: GBI, Glasgow Benefit Inventory; NOSE, Nasal Obstruction Septoplasty Effectiveness Questionnaire; postop6, 6 months postoperatively, postopo36, 36 months postoperatively; SD, standard deviation.

Note: ${ }^{\mathrm{a}}$ Kruskal-Wallis test.

relief was significantly lower among the patients who reported nasal obstruction at the long-term follow-up. ${ }^{16}$ These results are comparable to an earlier retrospective research by Dinis and Haider in which the authors mailed a questionnaire to 135 septoplasty patients to measure their satisfaction on a scale of 1 to 10 . They found that only $41.7 \%$ of the respondents reported a good to excellent result at the long-term ( 2 to 10 years) follow-up. ${ }^{17}$ In 1989 , Jessen et al, using a non-validated questionnaire, found that only $51 \%$ of their septoplasty patients reported subjective relief from nasal obstruction at 9 months, and only $26 \% 9$ years postoperatively, while the rates for "satisfied with the results" were of $74 \%$ at 9 months and of $69 \% 9$ years after surgery. They also used anterior rhinomanometry as an objective method for the measurement of the nasal resistance, and found that postoperatively the total nasal resistance was significantly more reduced at the nine-year follow-up when compared to the earlier one. ${ }^{20}$ These findings are similar to the results of the current study in the way that the symptom relief rate after septoplasty decreases with time. The symptom relief rates in our study are also better than those reported by Toyserkani and Frisch. In their retrospective questionnaire (based on the NOSE scale) study, $68 \%$ of the septoplasty patients experienced "improved nasal breathing" 11 years postoperatively, while $56 \%$ were satisfied with the overall outcome. Another finding that is in accordance with the current study's results was that patients with higher subjective improvements had higher satisfaction rates. ${ }^{18}$

Additionally, some authors have evaluated the long-term functional results of nasal septal surgery only by assessing the subjective improvement in specific nasal symptoms. ${ }^{21}$ Ghazipour et al, in their descriptive, prospective study, included 98 septoplasty patients who filled out the validated diseasespecific Sinonasal Outcome Test 20 (SNOT-20) questionnaire prior to the surgery. A great improvement in sinonasal headaches was found two years after the surgical procedure, as $83 \%$ of the patients reported complete recovery or pain reduction. The main limitation of the study was the gradual decline in the number of participants (35) in the long-term follow-up. ${ }^{21}$

In agreement with the current study, only a few existing studies have investigated the long-term changes in patients' QoL after nasal septal surgery. ${ }^{19,22-26}$ Schwentner et al also assessed the changes in the patients' general health and QoL after nasal septal surgery apart from nasal obstruction symptoms. This was a retrospective study that included answers from 285 patients who had undergone nasal septoplasty. They used a questionnaire based on the validated GBI and the Health-Related Quality of Life Questionnaire (HRQL) in rhino surgery. The questionnaire consisted of seven subscales: overall medical state, nasal symptoms, sleep, accompanying symptoms, practical problems, emotions, and social life. A significant amelioration in nasal symptoms, sleep, practical problems, and overall medical state was found seven years after surgery. However, no significant postoperative change was reported in the subgroups of emotion, social fife, and accompanying symptoms. $^{19}$

A difficulty when comparing the long-term outcome after septal surgery among the studies is that the surgical technique used can be different. Persicetti et al, for example, investigated the long-term outcomes of modified extracorporeal septoplasty in 120 patients. Rhinomanometric analyses and NOSE scores demonstrated statistically significant improvements in inspiratory flow and obstructive symptoms four to six years after surgery. In the group analysis, patients with severe and moderate septal deviations reported a more significant improvement in NOSE scores and in inspiratory flow when compared with patients with mild septal deviation. ${ }^{22}$

An ongoing discussion among rhinologists is whether it is reasonable to routinely perform objective measurements of nasal patency before and after surgery. The main argument against it is a huge discrepancy between the objective measurements and the subjective nasal obstruction found in many studies. ${ }^{17,24,27}$ However, it seems that the nasal airflow is significantly improved in the long-term after nasal septoplasty. This is in agreement with the findings of Haavisto and Sipilä, who reported that the AR measurements showed that nasal patency was significantly improved in a group of 30 septoplasty patients, while the amount of patients satisfied with the result (using a visual analogue score) was of $83 \% 10$ years after the operation. On the other hand, the rhinomanometry measurements demonstrated an increase in nasal resistance ten years postoperatively. ${ }^{23}$ Furthermore, Bohlin and Dahlqvist reported that the total nasal resistance, which was measured by rhinomanometry in a group of 35 septoplasty patients, was significantly reduced 10 years after surgery. ${ }^{28}$ According to the results of the current study, nasal patency (MCA values measured by AR) was significantly improved at all of the follow-up controls during the first three postoperative years. However, the changes in nasal obstruction symptoms had no significant 
correlation with the MCA values measured by AR. This result is in accordance with the report of Toyserkani et al. In this prospective study, patients with nasal obstruction symptoms due to nasal septum deviation underwent septoplasty and rated the improvement in their symptoms using the NOSE score eleven years postoperatively. Acoustic rhinometry was performed for the measurement of the nasal patency pre- and postoperatively. Total $56 \%$ of the patients reported subjective relief of nasal obstruction symptoms, and nasal patency was significantly improved at the long-term follow-up. However, the NOSE scores were not correlated with the AR measurements. ${ }^{24}$ Similarly, Dinis and Haider found that the correlation between preoperative nasal resistance (measured by rhinomanometry) and long-term postoperative success was not statistically significant. ${ }^{17}$ Taking into consideration the aforementioned findings, it seems that AR can show changes in volume and area inside the nasal cavity. However, the mechanism of nasal breathing is more complex and may be affected by additional factors. Therefore, AR can be used in the clinical research or as a supplementary objective method to examine the findings and the patients' symptom scores.

Nasal obstruction is the most common and significant preoperative symptom in patients who undergo nasal septoplasty. In the present study, the evaluation of nasal obstruction scores revealed a quite expected result: patients with greater improvements in nasal patency (higher MCA values) and nasal obstruction symptoms (better NOSE scores) had better QoL (better GBI results) postoperatively. However, this difference between the above and below the criteria groups was not statistically significant. On the other hand, considering the fact that the patients had a low mean GBI score $(<35 / 100)$, one could conclude that even the patients with postoperative improvement in nasal obstruction symptoms did not report very significant changes in their QoL. Similarly, in 2005, Konstantinidis et al investigated the long-term results of septoplasty in 67 patients. They used the Fairley Nasal Questionnaire (FNQ), which is a validated 12-item tool to measure nasal symptoms, and found a significant improvement in nasal obstruction 2 to 3 years after surgery. Moreover, patients with more significant improvement in nasal symptoms were more satisfied (higher GBI scores) with the result of the surgical procedure. However, $49 \%$ of the patients reported no satisfaction in the postoperative long-term, which probably indicates several unnecessary operations. Even in the group of satisfied patients, the mean total GBI score was of 23.8, which is low, like the score found in the current study. ${ }^{25}$ In 2007 , using the GBI, Calder and Swan found that nasal septoplasty resulted in benefits for 136 patients. The overall mean GBI score in their study was of 11.318 months after surgery. Despite the fact that this was a positive score, it suggested only a small change in the general health status. ${ }^{26}$ There are two possible explanations for this result. First of all, the GBI questions probably do not target those aspects of health-related QoL affected by nasal septoplasty. Secondly, nasal septal deviations cause minimal morbidity, and general health status questionnaires are therefore not the appropriate tools with which to measure the morbidity caused. Subsequently, a main result of the current study is that the GBI (QoL) scores three years after surgery were lower than those at the six-month follow-up, this time reaching statistical significance. That is due to the fact that subjective ratings of nasal symptom improvement and changes in the QoL in the short-term are mostly influenced by the placebo effect from the surgery, which is quite intense in the early postoperative period.

Although clinicians are considered very accurate in assessing the anatomic factors of a patient's nose, there are other parameters that contribute to the patient's disease-specific QoL besides the visible anatomy. ${ }^{17,20,25}$ This explains why the findings from AR measurements are difficult to interpret: they might accurately predict anatomic findings that, on the on the other hand, are not well correlated with nasal obstruction symptoms. Furthermore, the patients' perception of the nasal obstruction is more complex and may be affected by a variety of physiologic and psychological factors. The operative technique, an inappropriate indication for nasal septoplasty, ${ }^{11}$ the condition of the vascular and nerve supplies inside the nasal cavity and the expectations of the patients regarding the surgery may affect the perception of nasal obstruction and the outcome of the surgery. ${ }^{25}$ The coexistence of allergies or sinonasal disease with septal deviation is also reported to have a statistically significant association with higher rates of dissatisfaction after surgery, ${ }^{20}$ but in our study this was controlled, as it was one of the exclusion criteria. In conclusion, ENT surgeons need a reliable method to ascertain that their indication for septal surgery is appropriate, and the major problem is that there is no objective measurement of septal deviation. ${ }^{25}$

The strengths of the current study are its prospective and long-term design and the high follow-up rate (100\%). Additionally, the patients' short- and long-term benefits from nasal septoplasty were evaluated using objective and subjective methods. Furthermore, the operating physician was not personally collecting the follow-up outcome data from the patients, so the possibility of introducing a bias at that level was minimized. The clinical examination is subjective and may introduce an examination bias. Therefore, we used a validated instrument to achieve a patient-based outcome assessment. One weakness of the study design is the lack of a control group. However, there is no alternative treatment for a deviated nasal septum other than surgery. A nonsurgical control group could still be considered, however. Nevertheless, most patients desire to have their anatomic septum deviation surgically corrected, and we were concerned about being unable to recruit patients into a nonsurgical comparison group.

\section{Conclusion}

In patients with septal deformity, nasal septal surgery results in significant improvement in nasal obstruction symptoms, as well as in an increase in nasal patency. In the present study, the GBI scores reflected a statistically significant yet not important change in health status after septoplasty regarding the objectivity of the surgeons' criteria for nasal septoplasty. The study also indicates that the patients' positive evaluation of the septal surgery outcome tends to decrease with time. Further studies are needed to investigate the factors that affect the 
patients' QoL, and to establish valid selection criteria for patients eligible to undergo septoplasty, in order to reduce the number of unnecessary surgical interventions.

Conflicts of Interest

The authors have no conflicts of interest to disclose.

\section{References}

1 Kim CS, Moon BK, Jung DH, Min YG. Correlation between nasal obstruction symptoms and objective parameters of acoustic rhinometry and rhinomanometry. Auris Nasus Larynx 1998;25(01):45-48

2 Stewart MG, Smith TL, Weaver EM, et al. Outcomes after nasal septoplasty: results from the Nasal Obstruction Septoplasty Effectiveness (NOSE) study. Otolaryngol Head Neck Surg 2004; 130(03):283-290

3 Stewart MG, Witsell DL, Smith TL, Weaver EM, Yueh B, Hannley MT. Development and validation of the Nasal Obstruction Symptom Evaluation (NOSE) scale. Otolaryngol Head Neck Surg 2004; 130(02):157-163

4 Clement PA, Gordts F; Standardisation Committee on Objective Assessment of the Nasal Airway, IRS, and ERS. Consensus report on acoustic rhinometry and rhinomanometry. Rhinology 2005;43 (03):169-179

5 Arunachalam PS, Kitcher E, Gray J, Wilson JA. Nasal septal surgery: evaluation of symptomatic and general health outcomes. Clin Otolaryngol Allied Sci 2001;26(05):367-370

6 Siegel NS, Gliklich RE, Taghizadeh F, Chang Y. Outcomes of septoplasty. Otolaryngol Head Neck Surg 2000;122(02):228-232

7 Uppal S, Mistry H, Nadig S, Back G, Coatesworth A. Evaluation of patient benefit from nasal septal surgery for nasal obstruction. Auris Nasus Larynx 2005;32(02):129-137

8 Samad I, Stevens HE, Maloney A. The efficacy of nasal septal surgery. J Otolaryngol 1992;21(02):88-91

9 Pannu KK, Chadha S, Kaur IP. Evaluation of benefits of nasal septal surgery on nasal symptoms and general health. Indian J Otolaryngol Head Neck Surg 2009;61(01):59-65

10 Buckland JR, Thomas S, Harries PG. Can the Sino-nasal Outcome Test (SNOT-22) be used as a reliable outcome measure for successful septal surgery? Clin Otolaryngol Allied Sci 2003;28(01):43-47

11 Akduman D, Yanılmaz M, Haksever M, Doner F, Sayar Z. Patients' evaluation for the surgical management of nasal obstruction. Rhinology 2013;51(04):361-367

12 Manestar D, Braut T, Kujundžić M, et al. The effects of disclosure of sequential rhinomanometry scores on post-septoplasty subjective scores of nasal obstruction: a randomised controlled trial. Clin Otolaryngol 2012;37(03):176-180
13 Jin HR, Lee JY, Jung WJ. New description method and classification system for septal deviation. J Rhinol 2007;14(01):27-31

14 Lachanas VA, Tsiouvaka S, Tsea M, Hajiioannou JK, Skoulakis CE. Validation of the nasal obstruction symptom evaluation (NOSE) scale for Greek patients. Otolaryngol Head Neck Surg 2014;151 (05):819-823

15 Konstantinidis I, Triaridis S, Printza A, Triaridis A, Noussios G, Karagiannidis K. Assessment of patient benefit from septo-rhinoplasty with the use of Glasgow Benefit Inventory (GBI) and Nasal Symptom Questionnaire (NSQ). Acta Otorhinolaryngol Belg 2003;57(02):123-129

16 Sundh C, Sunnergren O. Long-term symptom relief after septoplasty. Eur Arch Otorhinolaryngol 2015;272(10):2871-2875

17 Dinis PB, Haider H. Septoplasty: long-term evaluation of results. Am J Otolaryngol 2002;23(02):85-90

18 Toyserkani NM, Frisch T. Are too many septal deviations operated on? A retrospective patient's satisfaction questionnaire with 11 years follow-up. Rhinology 2012;50(02):185-190

19 Schwentner I, Dejakum K, Schmutzhard J, Deibl M, Sprinzl GM. Does nasal septal surgery improve quality of life? Acta Otolaryngol 2006;126(07):752-757

20 Jessen M, Ivarsson A, Malm L. Nasal airway resistance and symptoms after functional septoplasty: comparison of findings at 9 months and 9 years. Clin Otolaryngol Allied Sci 1989;14(03):231-234

21 Ghazipour A, Abshirini H, Hekmat Shoar M, Pursalehan S. Sinonasal headaches and post-operative outcomes after septoplasty in patients with nasal septal deviation. Iran J Otorhinolaryngol 2011;23(65):133-139

22 Persichetti P, Toto V, Segreto F, Signoretti M, Marangi GF. Modified Extracorporeal Septoplasty: Functional Results at 6-Year Followup. Ann Plast Surg 2016;76(05):504-508

23 Haavisto LE, Sipilä JI. Acoustic rhinometry, rhinomanometry and visual analogue scale before and after septal surgery: a prospective 10-year follow-up. Clin Otolaryngol 2013;38(01):23-29

24 Toyserkani NM, Frisch T, Von Buchwald C. Postoperative improvement in acoustic rhinometry measurements after septoplasty correlates with long-term satisfaction. Rhinology 2013;51(02):171-175

25 Konstantinidis I, Triaridis S, Triaridis A, Karagiannidis K, Kontzoglou G. Long term results following nasal septal surgery. Focus on patients' satisfaction. Auris Nasus Larynx 2005;32(04):369-374

26 Calder NJ, Swan IRC. Outcomes of septal surgery. J Laryngol Otol 2007;121(11):1060-1063

27 André RF, Vuyk HD, Ahmed A, Graamans K, Nolst Trenité GJ. Correlation between subjective and objective evaluation of the nasal airway. A systematic review of the highest level of evidence. Clin Otolaryngol 2009;34(06):518-525

28 Bohlin L, Dahlqvist A. Nasal airway resistance and complications following functional septoplasty: a ten-year follow-up study. Rhinology 1994;32(04):195-197 